\title{
Dose-Adjusted EPOCH-R Compared With R-CHOP as Frontline Therapy for Diffuse Large B-Cell Lymphoma: Clinical Outcomes of the Phase III Intergroup Trial Alliance/CALGB 50303
}

\begin{abstract}
Nancy L. Bartlett, MD; Wyndham H. Wilson, MD, PhD²; Sin-Ho Jung, $\mathrm{PhD}^{3}$; Eric D. Hsi, MD; Matthew J. Maurer, MS ${ }^{5}$;

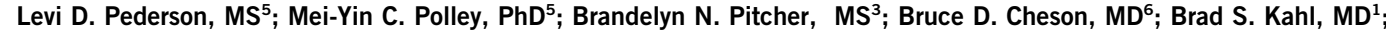

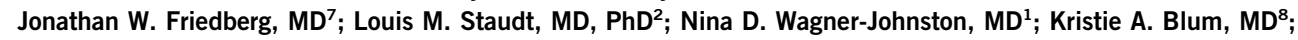
Jeremy S. Abramson, MD ${ }^{9}$; Nishitha M. Reddy, $\mathrm{MD}^{10}$; Jane N. Winter, MD ${ }^{11}$; Julie E. Chang, MD ${ }^{12}$; Ajay K. Gopal, MD ${ }^{13}$; Amy Chadburn, MD ${ }^{14}$; Susan Mathew, PhD ${ }^{14}$; Richard I. Fisher, MD ${ }^{15}$; Kristy L. Richards, MD, PhD ${ }^{16}$; Heiko Schöder, MD ${ }^{17}$; Andrew D. Zelenetz, MD, PhD ${ }^{17}$; and John P. Leonard, MD ${ }^{14}$
\end{abstract}

ASSOCIATED CONTENT

Listen to the podcast

by Dr Stiff at ascopubs.org/jco/ podcasts

Data Supplements

Author affiliations

and support

information (if

applicable) appear at the end of this article.

Accepted on February 27, 2019 and published at jco.org on April 2, 2019:

DOI https://doi.org/10. 1200/JC0.18.01994

Processed as a Rapid Communication manuscript.

Clinical trial

information:

NCT00118209.

The content is solely the responsibility of

the authors and does not necessarily

represent the official views of the National Institutes of Health.

The study was coordinated by Alliance/Cancer and Leukemia Group B (CALGB).

Participating groups included Eastern

Cooperative

Oncology Group,

North Central Cancer

Treatment Group,

National Surgical

Adjuvant Breast and

Bowel Project,

and SWOG.

PURPOSE Alliance/CALGB 50303 (NCT00118209), an intergroup, phase III study, compared dose-adjusted etoposide, prednisone, vincristine, cyclophosphamide, doxorubicin, and rituximab (DA-EPOCH-R) with standard rituximab, cyclophosphamide, doxorubicin, vincristine, and prednisone (R-CHOP) as frontline therapy for diffuse large B-cell lymphoma.

PATIENTS AND METHODS Patients received six cycles of DA-EPOCH-R or R-CHOP. The primary objective was progression-free survival (PFS); secondary clinical objectives included response rate, overall survival (OS), and safety.

RESULTS Between 2005 and 2013, 524 patients were registered; 491 eligible patients were included in the final analysis. Most patients (74\%) had stage III or IV disease; International Prognostic Index (IPI) risk groups included $26 \%$ IPI 0 to $1,37 \%$ IPI 2, 25\% IPI 3, and 12\% IPI 4 to 5 . At a median follow-up of 5 years, PFS was not statistically different between the arms (hazard ratio, $0.93 ; 95 \% \mathrm{Cl}, 0.68$ to $1.27 ; P=.65$ ), with a 2-year PFS rate of $78.9 \%(95 \% \mathrm{Cl}, 73.8 \%$ to $84.2 \%)$ for DA-EPOCH-R and $75.5 \%(95 \% \mathrm{Cl}, 70.2 \%$ to $81.1 \%)$ for R-CHOP. OS was not different (hazard ratio, $1.09 ; 95 \% \mathrm{Cl}, 0.75$ to $1.59 ; P=.64)$, with a 2 -year OS rate of $86.5 \%(95 \% \mathrm{Cl}$, $82.3 \%$ to $91 \%$ ) for DA-EPOCH-R and $85.7 \%$ (95\% Cl, 81.4\% to 90.2\%) for R-CHOP. Grade 3 and 4 adverse events were more common $(P<.001)$ in the DA-EPOCH-R arm than the R-CHOP arm, including infection (16.9\% $\vee 10.7 \%$, respectively), febrile neutropenia (35.0\% $\vee 17.7 \%$, respectively), mucositis $(8.4 \% \vee 2.1 \%$, respectively), and neuropathy (18.6\% $\vee 3.3 \%$, respectively). Five treatment-related deaths $(2.1 \%)$ occurred in each arm.

CONCLUSION In the 50303 study population, the more intensive, infusional DA-EPOCH-R was more toxic and did not improve PFS or OS compared with R-CHOP. The more favorable results with R-CHOP compared with historical controls suggest a potential patient selection bias and may preclude generalizability of results to specific risk subgroups.

J Clin Oncol 37:1790-1799. $\odot 2019$ by American Society of Clinical Oncology

\section{INTRODUCTION}

In 2002, the first of three trials established rituximab (R) plus CHOP (cyclophosphamide, doxorubicin, vincristine, prednisone; R-CHOP refers to the combination regimen) as frontline standard of care for diffuse large B-cell lymphoma (DLBCL). ${ }^{1-5}$ The 3-year event-free survival (EFS) rate ranged from $53 \%$ in patients age 60 years or older with high-risk features to $79 \%$ in patients 18 to 60 years old with a low-risk International Prognostic Index (IPI). ${ }^{3,4}$ Less favorable outcomes for patients with recurrent $\mathrm{DLBCL}^{6}$ prompted efforts to improve first-line approaches and biomarkers to identify high-risk patients.
National Cancer Institute ( $\mathrm{NCl}$ ) investigators modified the $\mathrm{CHOP}$ regimen and developed the 96-hour infusional dose-adjusted (DA) etoposide, prednisone, vincristine, cyclophosphamide, and doxorubicin (EPOCH) combination. Rationale included evidence of less tumor resistance with prolonged exposure to natural products, less cardiac toxicity with prolonged doxorubicin administration, and maximization of dose intensity by pharmacodynamic dose adjustment on the basis of each cycle's neutrophil nadir. 7,8 The initial DA-EPOCH study in untreated DLBCL reported a 62-month progression-free survival (PFS) rate of 70\% and overall survival (OS) rate of $73 \%$, better results than 
with $\mathrm{CHOP} .{ }^{8}$ Rituximab was added to DA-EPOCH, resulting in a 12-month PFS rate of $85 \%{ }^{9,10} \mathrm{~A}$ phase II, multicenter trial of DA-EPOCH-R in DLBCL by Cancer and Leukemia Group B (CALGB) confirmed the regimen could be safely and accurately administered in community settings, with outcomes (5-year time-to-progression rate, 81\%) similar to $\mathrm{NCl}$ data. ${ }^{11}$ A phase III trial comparing R-CHOP with DA-EPOCH-R in frontline therapy of DLBCL (a collaboration between the $\mathrm{NCl}$ and the US Intergroup) was coordinated by CALGB (now part of the Alliance for Clinical Trials) and activated in 2005. We report clinical outcomes with a median follow-up of 5 years. Additional primary and secondary objectives will be reported separately.

\section{PATIENTS AND METHODS}

\section{Study Design and Patients}

Alliance/CALGB 50303 compared DA-EPOCH-R with $\mathrm{R}-\mathrm{CHOP}$ in a 1:1 randomization. Eligible patients included untreated DLBCL (including morphologic variants centroblastic, immunoblastic, T-cell/histiocyte rich, and anaplastic), primary mediastinal large B-cell lymphoma (PMBCL), and intravascular large B-cell lymphoma confirmed by central pathology review. Patients with underlying indolent lymphoma in the bone marrow or in a biopsy specimen were excluded. Before registration, limited field radiation or fewer than 10 days of glucocorticoid treatment for urgent disease complications (eg, cord compression) were allowed. Additional eligibility criteria included age at least 18 years, stage II to IV DLBCL (or stage I PMBCL), Eastern Cooperative Oncology Group performance status 0 to 2, left ventricular ejection fraction rate greater than $45 \%$, absolute neutrophil count (ANC) greater than $1,000 / \mu \mathrm{L}$, platelet count greater than $100,000 / \mu \mathrm{L}$, creatinine level not more than $1.5 \mathrm{mg} / \mathrm{dL}$, and bilirubin level not more than $2 \mathrm{mg} / \mathrm{dL}$. Patients with known CNS disease or HIV were excluded.

A fresh (frozen) tumor biopsy specimen was required before registration. When necessary, a repeated study biopsy (excisional or core needle) was required, unless not feasible (and exception granted by study chairs). If fresh tissue was unavailable, stained and unstained slides or blocks were requested. Tissue was prioritized for primary and secondary molecular correlates and for central pathology review. Immunohistochemistry for BCL-2 (DAKO 124; Agilent, Santa Clara, CA) and MYC (Ventana Y69; Roche, Tucson, AZ) expression and fluorescence in situ hybridization (FISH) testing for MYC, BCL2, and BCL6 rearrangements were not preplanned but were performed centrally on patients with available material.

Participants provided institutional review board-approved informed consent in accordance with federal and institutional guidelines. Data collection and statistical analyses were conducted by the Alliance Statistics and Data Center. Data quality was ensured through data review by the Alliance Statistics and Data Center and the study chairperson following Alliance policies.

\section{Treatment}

R-CHOP and DA-EPOCH-R administration was planned every 21 days for six cycles. Dosing, escalation and deescalation of DA-EPOCH-R on the basis of nadir ANC and platelet count are as previously reported ${ }^{8}$ and described in the Data Supplement for this report. Patients with more than one extranodal site and elevated lactate dehydrogenase level, or bone marrow involvement were to receive CNS prophylaxis with intrathecal methotrexate, $12 \mathrm{mg}$ on day 1 or day 2 of cycles 3 through 6 . Consolidative radiotherapy (RT) was prohibited. Required ancillary medications for both regimens included a proton pump inhibitor, stool softeners, and, for patients positive for hepatitis B surface antigen, lamivudine $100 \mathrm{mg}$ per day. Pneumocystis prophylaxis was required with DA-EPOCH-R. Filgrastim was required on days 6 through 15 of DAEPOCH-R or until ANC was greater than $5,000 / \mu \mathrm{L}$ after nadir; pegfilgrastim was allowed with study chair approval. In the R-CHOP arm, filgrastim or pegfilgrastim was initiated if a patient experienced an ANC of less than $500 / \mu \mathrm{L}$, febrile neutropenia with the previous cycle, or at physician discretion.

\section{Efficacy and Safety Measures}

The investigator determined response according to the 1999 International Working Group response criteria. ${ }^{12}$ End-of-treatment (EOT) positron emission tomography (PET)-computed tomography (CT) was recommended for patients with partial response, complete response (CR), or CR unconfirmed (CRu). Baseline, postcycle 2, and EOT PET were only required for patients participating in the optional imaging companion study (CALGB 580603). If an EOT PET was performed, either as standard of care or in the companion study, results were incorporated into the response determination with a negative PET-defined as uptake less than or equal to the mediastinal blood pool (Data Supplement). Adverse events were reported according to the revised $\mathrm{NCl}$ Common Terminology Criteria for Adverse Events, version 4.0.

\section{Statistical Analysis}

The primary clinical end point was PFS, measured from randomization to progression, relapse, or death from any cause, whichever occurred first. Secondary clinical objectives included comparisons of response rate, OS, and toxicity. The protocol required up to 5 years of follow-up. The initial sample size was 478, assuming a 3-year PFS of $55 \%$ in the R-CHOP arm, a hazard ratio (HR) of 0.65 for DA-EPOCH-R versus R-CHOP detectable with 90\% power, a two-sided $\alpha 0.05$ log-rank test, and a 10\% ineligibility rate. Sample size was increased to 523 owing to lower than expected participation in the companion imaging study, CALGB 580603. 
Final analysis was initially planned after 242 PFS events; however, as a result of significantly fewer events than predicted, the Data and Safety Monitoring Board recommended release of the data in July 2016, after 167 PFS events. All analyses were based on modified intent to treat, including eligible patients randomly assigned to treatment, and were performed with SAS, version 9.4. The primary end point was assessed between arms using unadjusted Cox models and Cox models stratified by IPI. Statistical tests reported were twosided and $P<.05$ was used to declare statistical significance for the primary end point. No planned clinical subset analyses were in the protocol and all analyses beyond the primary end points should be considered post hoc. Additional statistical details are included in the Data Supplement.

\section{RESULTS}

\section{Patients}

From 2005 to 2013, 524 patients were randomly assigned to R-CHOP or DA-EPOCH-R (Fig 1). Data cutoff occurred on November 11, 2017. Of 491 eligible patients (93.7\%), 250 were assigned to R-CHOP and 241 to DA-EPOCH-R. Median time from diagnosis to registration and initiation of therapy was 18 days and 21 days, respectively. Ten patients ( $n=6$ in the R-CHOP arm and $n=4$ in the DAEPOCH-R arm) never started the assigned treatment (one died before starting and nine refused or withdrew) but were included in the intent-to-treat analysis. One of five expert hematopathologists reviewed pathology centrally on 429 patients (82.0\%) and 18 (3.4\%) were excluded (Fig 1). The principal investigator reviewed diagnostic biopsy reports for all cases not centrally reviewed to confirm DLBCL. Fifteen patients $(2.9 \%)$ were excluded during case evaluations (Fig 1). The study arms were well balanced for baseline characteristics (Table 1; Data Supplement). Most patients had stage III or IV disease $(74.0 \%)$ with IPI risk group distribution as follows: 0 to $1,26.0 \% ; 2,37.0 \% ; 3$, $25.0 \%$; and 4 to $5,12.0 \%$. In terms of age, $19.0 \%$ of patients ( $n=93$ ) were at least 70 years old and $2.6 \%$ $(n=13)$ were 80 years or older. As allowed, seven patients received short-course local RT and 35 patients received corticosteroids briefly before initiating protocol therapy.

Double expressor (DE) status was determined centrally in 270 patients (55.0\%), of whom 42 patients (15.6\%) were classified as DE (BCL-2 $\geq 50 \%$ and $M Y C \geq 40 \%$ ). MYC, $B C L-2$, and $B C L-6$ rearrangements were determined centrally by FISH on available tissue. MYC rearrangement data were available on 249 patients (50.7\%), of whom 13 (5.2\%) were positive for rearrangements, six in the R-CHOP arm and seven in the DA-EPOCH-R arm. Of the 13 MYC rearranged cases, three had rearrangements of BCL-2 and/or BCL-6 (double hit) and 10 had incomplete DE data.

\section{Treatment}

All six treatment cycles were completed by $88.0 \%$ of the $\mathrm{R}-\mathrm{CHOP}$ and $82.0 \%$ of the DA-EPOCH-R group. Reasons for

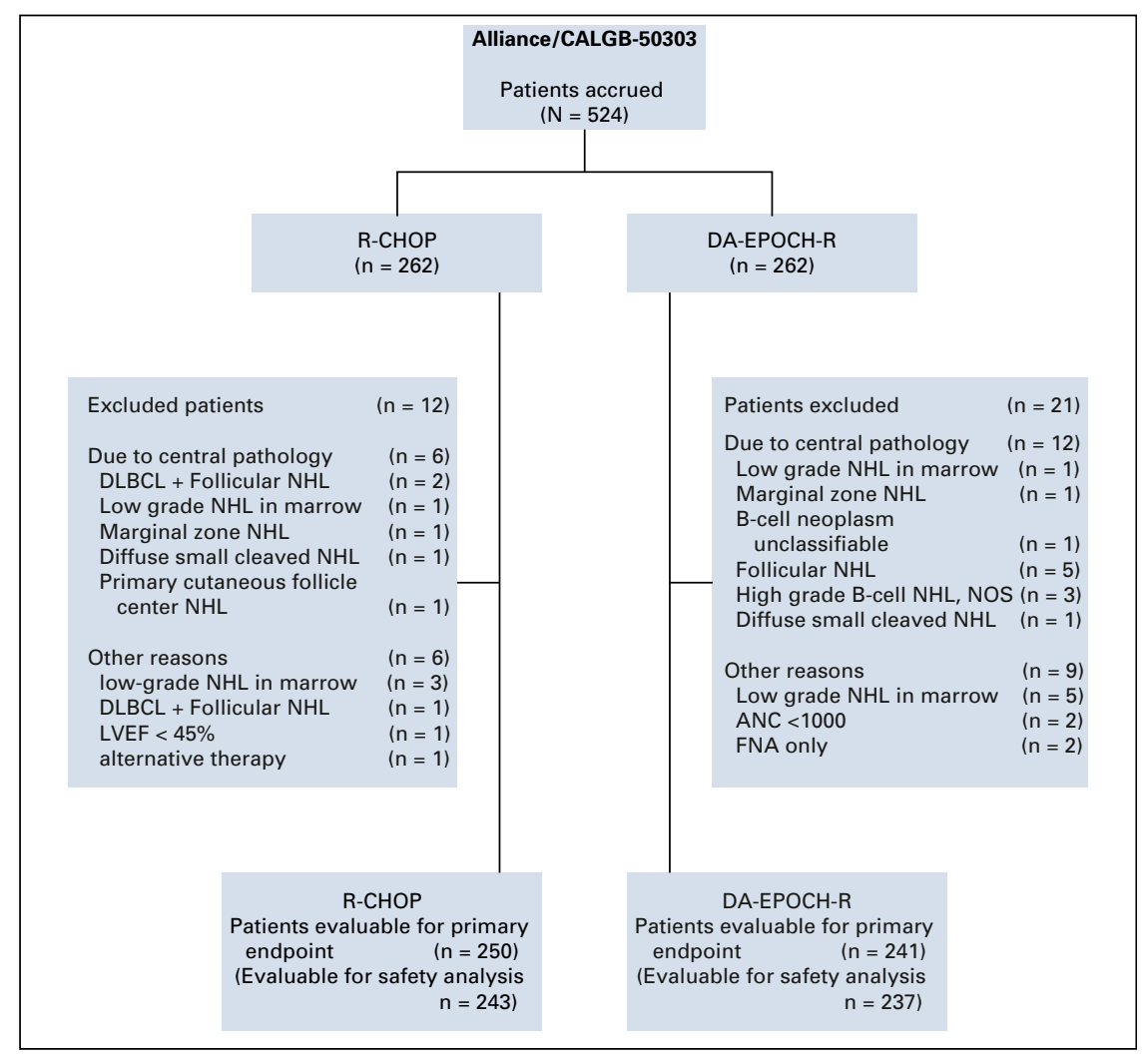

FIG 1. CONSORT diagram. ANC, absolute neutrophil count; CALGB, Cancer and Leukemia Group B; DA-EPOCH-R, dose-adjusted etoposide, prednisone, vincristine, cyclophosphamide, doxorubicin, and rituximab; DLBCL, diffuse large B-cell lymphoma; FNA, fine-needle aspirate; LVEF, left ventricular ejection fraction; NHL, non-Hodgkin lymphoma; NOS, not otherwise specified; R-CHOP, rituximab, cyclophosphamide, doxorubicin, vincristine, and prednisone. 
TABLE 1. Characteristics of Eligible Patients

\begin{tabular}{|c|c|c|c|}
\hline \multirow[b]{2}{*}{ Characteristic } & \multicolumn{2}{|c|}{ Treatment Received } & \multirow[b]{2}{*}{$P$} \\
\hline & $\begin{array}{c}\text { R-CHOP } \\
(n=250)\end{array}$ & $\begin{array}{c}\text { DA-EPOCH-R } \\
(n=241)\end{array}$ & \\
\hline Sex & & & $.7630 \dagger$ \\
\hline Missing & 1 & 0 & \\
\hline Male & $133(53.4)$ & $132(54.8)$ & \\
\hline Female & $116(46.6)$ & $109(45.2)$ & \\
\hline Age, years & & & $.8567^{*}$ \\
\hline No. (no. missing) & $247(3)$ & $239(2)$ & \\
\hline Median & 58.0 & 58.0 & \\
\hline Range & $18.0-86.0$ & $19.0-84.0$ & \\
\hline Age group, years & & & $.9579 \dagger$ \\
\hline Missing & 3 & 2 & \\
\hline $18-60$ & $141(57.1)$ & $137(57.3)$ & \\
\hline$>60$ & $106(42.9)$ & $102(42.7)$ & \\
\hline ECOG PS & & & $.2069 \dagger$ \\
\hline Missing & 1 & 0 & \\
\hline 0 & $101(40.6)$ & $113(46.9)$ & \\
\hline 1 & $119(47.8)$ & $96(39.8)$ & \\
\hline 2 & 29 (11.6) & 32 (13.3) & \\
\hline Extranodal disease ( $\geq 1$ site) & $64(25.7)$ & $68(28.2)$ & $.5308 \dagger$ \\
\hline Stage & & & $.6654 \dagger$ \\
\hline Missing & 7 & 5 & \\
\hline I & $7(2.9)$ & $6(2.5)$ & \\
\hline II & $53(21.8)$ & $48(20.3)$ & \\
\hline III & $71(29.2)$ & $60(25.4)$ & \\
\hline IV & $112(46.1)$ & $122(51.7)$ & \\
\hline IPI risk group & & & $.6047 \dagger$ \\
\hline Missing & 9 & 6 & \\
\hline Low & $64(26.6)$ & $59(25.1)$ & \\
\hline Low-intermediate & 93 (38.6) & $83(35.3)$ & \\
\hline High-intermediate & $60(24.9)$ & $61(26.0)$ & \\
\hline High & $24(10.0)$ & 32 (13.6) & \\
\hline
\end{tabular}

NOTE. Data reported as No. (\%) unless otherwise indicated.

Abbreviations: DA-EPOCH-R, dose-adjusted etoposide, prednisone, vincristine, cyclophosphamide, doxorubicin, and rituximab; DLBCL, diffuse large B-cell Iymphoma; ECOG, Eastern Cooperative Oncology Group; IPI, International Prognostic Index; PS, performance status; R-CHOP, rituximab, cyclophosphamide, doxorubicin, vincristine, and prednisone.

*Kruskal-Wallis test.

$\dagger \chi^{2}$ test.

early discontinuation included disease progression (R-CHOP, 2.8\%; DA-EPOCH-R, 1.3\%), adverse events (R-CHOP, 2.0\%; DA-EPOCH-R, 6.3\%), death (R-CHOP, $1.6 \%$; DA-EPOCH-R, 2.5\%), patient withdrawal after initiating treatment (R-CHOP, 1.2\%; DA-EPOCH-R, 2.9\%), patient withdrawal before beginning protocol treatment (R-CHOP, 2.4\%; DA-EPOCH-R, 1.7\%), alternative therapy
(R-CHOP, 0.8\%; DA-EPOCH-R, 1.3\%), and other or missing reasons (R-CHOP, 1.6\%; DA-EPOCH-R, 2.9\%). The maximum dose level (DL) achieved with DA-EPOCH-R was DL1 in $25.3 \%$, DL2 in $19.8 \%$, DL3 in $24.5 \%$, DL4 in $16.5 \%$, and greater than DL4 in $13.9 \%$. Intrathecal methotrexate prophylaxis was administered in $19.8 \%$ of the R-CHOP group and $27.0 \%$ of the DA-EPOCH-R group $(P=.060)$. Of patients in the R-CHOP group, $64.2 \%$ received growth factor support at least once, including 54 (22.2\%) who initiated filgrastim with cycle 1.

Efficacy. Across arms, at median follow-up of 5.2 years (interquartile range, 4.8-5.4), 159 patients had a PFS event and 109 patients died. There was no statistically significant difference in PFS between arms (DA-EPOCH-R HR, 0.93, 95\% Cl, 0.68 to 1.27; $P=.65$; Fig 2). Two-year and 5-year PFS rates for R-CHOP were $75.5 \%$ and $66.0 \%$, respectively, and for DA-EPOCH-R, $78.9 \%$ and $68.0 \%$ respectively (Table 2). These results were consistent when stratified by IPI (DA-EPOCH-R HR, 0.84; 95\% Cl, 0.61 to 1.15; $P=.27$ ). OS was similar (DA-EPOCH-R HR, 1.09, 95\% Cl, 0.75 to 1.59; $P=.64$; Fig 3) with 5-year OS rates of $78.5 \%$ for $\mathrm{R}-\mathrm{CHOP}$ and $77.5 \%$ for DA-EPOCH-R. The overall response rate was $88.0 \%$ (CR/Cru, 59.6\%) in the R-CHOP group and $86.7 \%$ (CR/Cru, 58.5\%) in the DA-EPOCH-R group ( $P=.67$ ).

The 2- and 5-year PFS rates for all patients was 77.1\% $(95 \% \mathrm{Cl}, 73.5$ to 81$)$ and $67.1 \%(95 \% \mathrm{Cl}, 62.8$ to 71.6$)$, respectively, with a 5-year OS rate of $78.0 \%(95 \% \mathrm{Cl}, 74.3$ to 81.9). Patients older than 60 years had inferior PFS compared with younger patients ( $\mathrm{HR}, 1.43 ; 95 \% \mathrm{Cl}, 1.04$ to $1.95 ; P=.026)$ with a 2 -year PFS rate of $75.2 \%(95 \% \mathrm{Cl}$, 69.5 to 81.4 ) versus $78.9 \%(95 \% \mathrm{Cl}, 74.2$ to 83.9$)$, respectively, and 5 -year PFS rate of $62 \%(95 \% \mathrm{Cl}, 55.5$ to 69.2 ) versus $71.3 \%$ (95\% Cl, 65.9 to 77.2$)$, respectively. CNS relapse occurred in 18 patients (3.7\%); six had received intrathecal prophylaxis. PFS was strongly associated with IPI $(P<.001)$; the 2-year PFS rate was $91.7 \%(95 \%$ $\mathrm{Cl}, 86.9$ to 96.8$)$ for IPI 0 to $1 ; 76.5 \%(95 \% \mathrm{Cl}, 70.4$ to 83.2$)$ for IPI 2; 70.7\% (95\% Cl, 63 to 79.4 ) for IPI 3; and $62.2 \%$ ( $95 \% \mathrm{Cl}, 50.7$ to 76.4 ) for IPI 4 to 5 (Data Supplement). Among 270 patients with DE data, PFS was inferior (HR, $1.75 ; 95 \% \mathrm{Cl}, 1.03$ to $2.98 ; P=.037)$ for DE-positive patients $(n=42)$ compared with 228 DE-negative patients (Data Supplement).

Subgroup analysis. Figure 4 shows the post hoc comparison of PFS by arm in subgroups of standard clinical features, including age, lactate dehydrogenase level, Eastern Cooperative Oncology Group performance status, extranodal disease, stage, and IPI risk group. No adjustment for multiple comparisons is reported for these post hoc analyses and the study was not powered for these comparisons. PFS was higher in the DA-EPOCH-R arm in the highest risk IPI (ie, IPI 4 to 5) subgroup (HR, 0.46; $95 \% \mathrm{CI}$, 0.21 to 1.01; unadjusted $P=.052$ ) as well as when grouping patients with IPI 3 to 5 together (HR, 0.63, 95\% $\mathrm{Cl}, 0.41$ to 0.99, unadjusted $P=.041$; Data Supplement). 


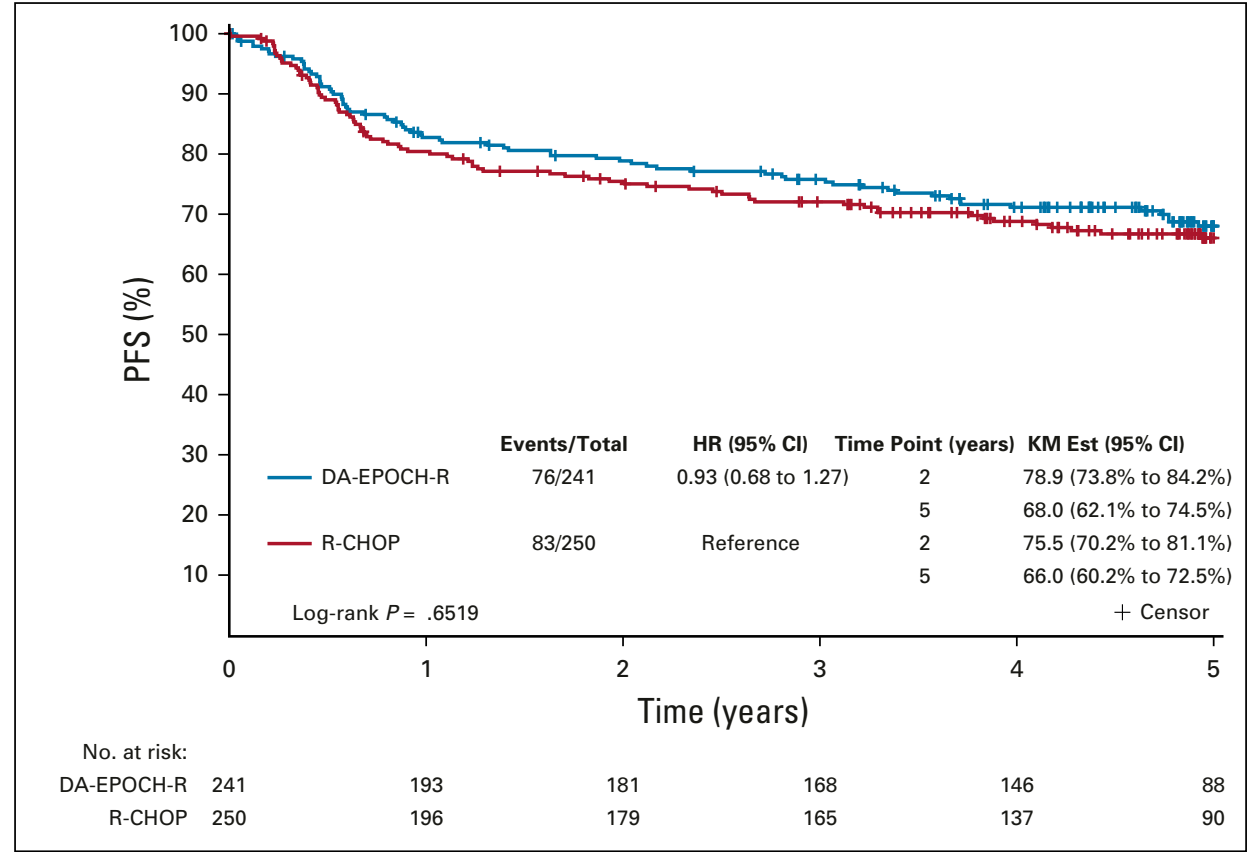

FIG 2. Progression-free survival (PFS) by treatment arm. Kaplan-Meier PFS estimate of all patients by treatment arm in years since random assignment. There was no statistically significant difference in PFS between arms ( $P=$.6519). DAEPOCH-R, dose-adjusted etoposide, prednisone, vincristine, cyclophosphamide, doxorubicin, and rituximab; HR, hazard ratio; KM Est, KM, Kaplan-Meier estimate; R-CHOP, rituximab, cyclophosphamide, doxorubicin, vincristine, and prednisone.

However, no difference in OS was observed in this subgroup (Data Supplement). No meaningful difference in PFS or OS between arms for any of the other subgroups was demonstrated.

CNS relapse occurred in $4.0 \%$ of patients $(n=10)$ treated with R-CHOP and 3.3\% $(n=8)$ treated with DA-EPOCH-R. Compliance with protocol-specified CNS prophylaxis was not captured precisely. However, there was no clear impact of prophylaxis on CNS relapse in our limited data set (Data Supplement).

Among 35 patients with PMBCL, PFS events occurred in three of 20 patients treated with R-CHOP and two of
15 patients treated with DA-EPOCH-R. No patients with $P M B C L$ received $R T$. There was no difference in PFS or OS for 42 patients with DE phenotype who received R-CHOP $(n=22)$ versus DA-EPOCH-R ( $n=20$; Data Supplement). Progression or death occurred in seven of 13 patients with MYC rearrangement: four of seven treated with DA-EPOCH$\mathrm{R}$ and three of six treated with R-CHOP.

Safety. The safety analysis population comprised eligible patients with available toxicity data $(n=480)$. There were five $(2.1 \%)$ treatment-related deaths per arm (causes in RCHOP arm: infection [ $n=2]$, cardiac [ $n=1]$, CNS hemorrhage $[n=1]$, unknown $[n=1]$; causes in DA-EPOCH-R

TABLE 2. Progression-Free Survival for All Patients and According to Treatment Arm

Parameter

Event/Total

Hazard Ratio $(95 \% \mathrm{CI})^{*}$

\begin{tabular}{|c|c|c|c|c|}
\hline raralineter & Eveliviutal & nazalu katio (95\% (I) & Suivival Estimátes (95\% CI), \% & \\
\hline & & & 5 Years: 67.1 (62.8 to 71.6$)$ & \\
\hline Treatment arm & & & & $.6519 \ddagger$ \\
\hline \multirow[t]{2}{*}{ DA-EPOCH-R } & $76 / 241$ & 0.93 (0.68 to 1.27$)$ & 2 Years: 78.9 (73.8 to 84.2) & \\
\hline & & & 3 Years: 75.8 (70.5 to 81.5) & \\
\hline \multirow[t]{3}{*}{$\mathrm{R}-\mathrm{CHOP}$} & $83 / 250$ & Reference & 2 Years: 75.5 (70.2 to 81.1 ) & \\
\hline & & & 3 Years: 72.0 (66.6 to 77.9$)$ & \\
\hline & & & 5 Years: 66.0 (60.2 to 72.5$)$ & \\
\hline
\end{tabular}

Abbreviations: DA-EPOCH-R, dose-adjusted etoposide, prednisone, vincristine, cyclophosphamide, doxorubicin, and rituximab; R-CHOP, rituximab, cyclophosphamide, doxorubicin, vincristine, and prednisone.

${ }^{*}$ Cox model.

†Kaplan-Meier method.

łLog-rank test. 


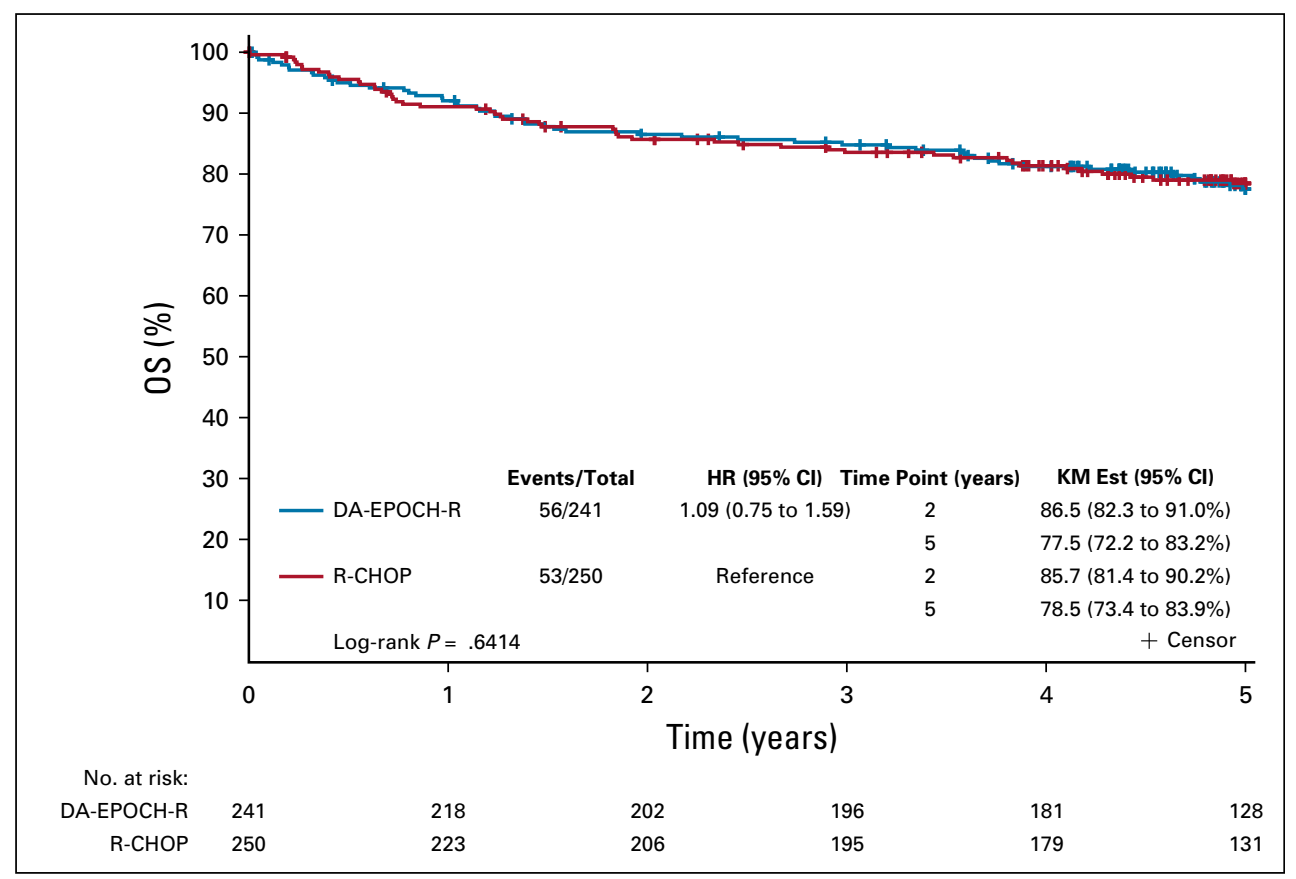

FIG 3. Overall survival (OS) by treatment arm. KaplanMeier OS estimate of all patients by treatment arm in years since random assignment. The 5-year OS was similar between the R-CHOP and DA-EPOCH-R arms $(P=.6414)$. DA-EPOCH-R, dose-adjusted etoposide, prednisone, vincristine, cyclophosphamide, doxorubicin, and rituximab; HR, hazard ratio; KM Est, KM, Kaplan-Meier estimate; R$\mathrm{CHOP}$, rituximab, cyclophosphamide, doxorubicin, vincristine, and prednisone.

arm: infection [ $n=2]$, cardiac $[n=1]$, sudden death $[n=1]$, multiorgan failure $[n=1])$. Grade 3 to 5 treatmentrelated events occurred in $98.3 \%$ of patients in the DAEPOCH-R arm and $78.2 \%$ in the R-CHOP arm $(P<.001)$. Overall grade 3 to 4 , treatment-related hematologic $(97.5 \% \vee 73.7 \% ; P<.001)$ and nonhematologic $(72.2 \%$ $v 43.2 \% ; P<.001)$ adverse events were more frequent in the DA-EPOCH-R arm. Table 3 lists selected toxicities that the investigator deemed at least possibly related to treatment and were significantly different between arms. Late cardiac events occurred in six patients in the RCHOP arm ( $n=5$ left ventricular systolic dysfunction, $n=$ 1 atrial fibrillation) and two patients in the DA-EPOCH-R arm ( $n=1$ myocardial infarction with heart failure, $n=1$ atrial fibrillation). Two patients receiving DA-EPOCH-R and one receiving $\mathrm{R}-\mathrm{CHOP}$ died of secondary acute myeloid leukemia.

\section{DISCUSSION}

This randomized trial compared the efficacy of R-CHOP to the more intensive, infusional DA-EPOCH-R in patients with untreated DLBCL. Despite greater toxicity and complexity, there was no improvement in PFS, OS, or response rate with DA-EPOCH-R. In post hoc subset analyses, patients with IPI 3 to 5 who received DAEPOCH-R had improved PFS ( $\mathrm{HR}=0.63,95 \% \mathrm{Cl}: 0.41$ to 0.99; unadjusted $P=0.041$ ) compared with patients in the R-CHOP arm; however, the subset analysis was unplanned and not powered, and the significance of the unadjusted $P$ value must be tempered in light of multiple comparisons. There was no difference between arms in
OS (HR, 0.78; 95\% Cl, 0.47 to 1.32 ; unadjusted $P=.36$ ) in this subgroup.

The trial design of Alliance/CALGB 50303 assumed a 55\% 3-year PFS rate in the R-CHOP arm and targeted a HR of 0.65 for DA-EPOCH-R compared with R-CHOP. The observed 3-year PFS rate for R-CHOP in our study was significantly better, at $72 \%(95 \% \mathrm{Cl}, 66.6 \%$ to $77.9 \%$ ). By comparison, in the broad-based population cohort of the Mayo/lowa Molecular Epidemiology Resource, the 3-year EFS rate was $61 \%(95 \% \mathrm{Cl}, 57 \%$ to $64 \%$ ) for 686 patients with stage II to IV DLBCL or PMBCL treated with R-CHOP, suggesting enrollment of patients with better outcomes than similar IPI risk-stratified patients outside of trials. ${ }^{13}$ The median time from diagnosisto-treatment initiation was 21 days for Alliance/CALBG 50303 versus 15 in the Mayo/lowa Molecular Epidemiology Resource study; longer diagnosis-to-treatment initiation is associated with more favorable clinical characteristics and better outcomes, and may reflect under enrollment of higher-risk patients (ie, poor performance status, rapidly progressive disease) in the 50303 trial, because of the inability to delay treatment to meet trial enrollment requirements. ${ }^{14}$ Furthermore, the requirement for fresh tissue on the 50303 trial for molecular profiling may have exacerbated this effect. Other prospective trials have reported similar issues, such as the PYRAMID trial (NCT00931918) in patients with nongerminal center B-cell-like DLBCL, with a 2-year PFS rate with $\mathrm{R}-\mathrm{CHOP}$ of $78 \%$, compared with the historically reported $30 \%$ to $40 \%$ in this subgroup. ${ }^{15,16}$ This unintended enrichment for favorable patients could mask any benefit of a new combination in higher-risk patients. 


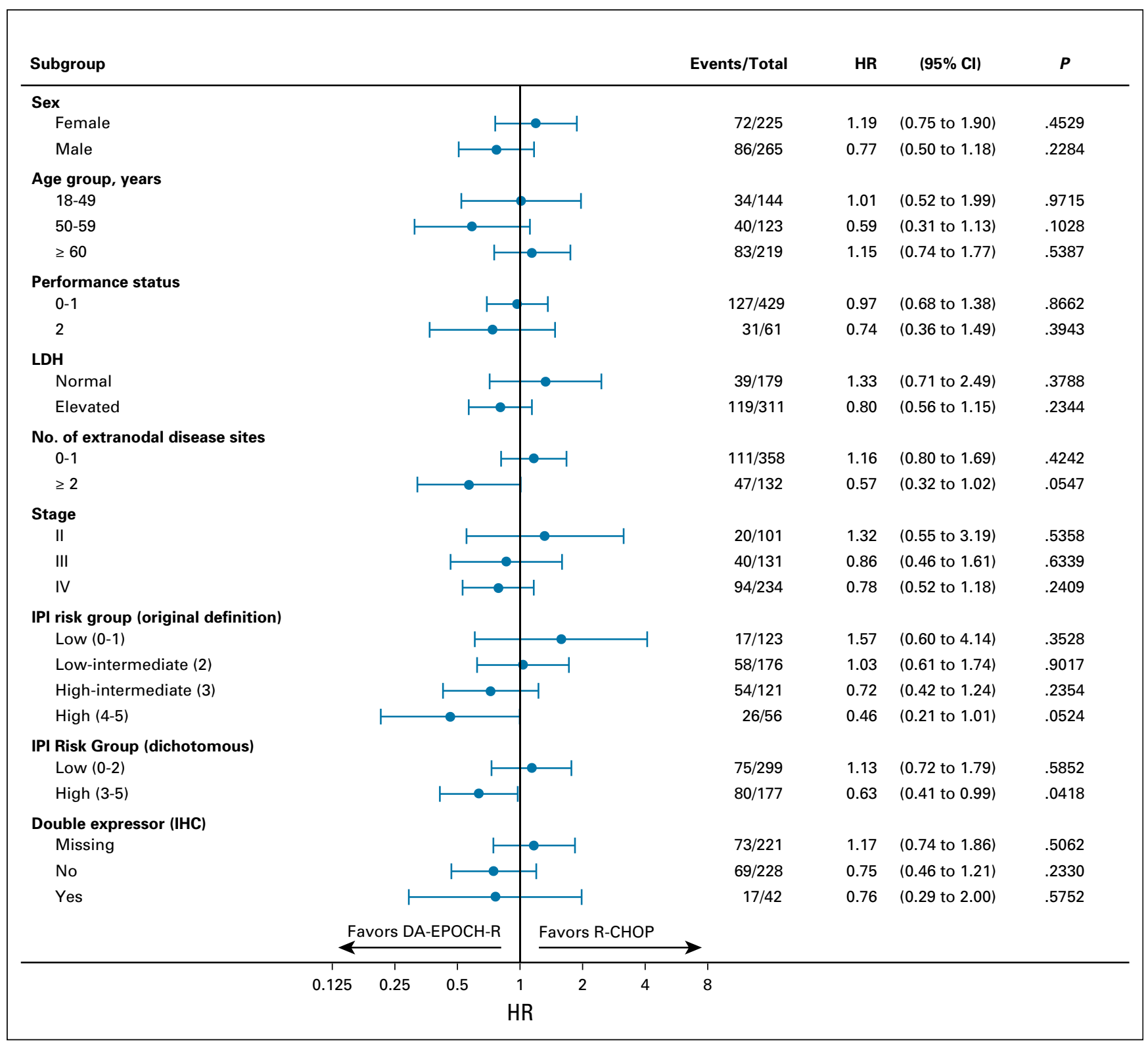

FIG 4. Progression-free survival (PFS) by subset analysis. Post hoc comparison of PFS by arm in subgroups of standard clinical features, including age, LDH level, Eastern Cooperative Oncology Group performance status, extranodal disease, stage, and IPI risk group. PFS was higher in the DA-EPOCH-R arm in the highest risk IPI (4-5) subgroup (unadjusted $P=.0524$ ). No meaningful difference in PFS between arms for any of the other subgroups was demonstrated. DA-EPOCH-R, dose-adjusted etoposide, prednisone, vincristine, cyclophosphamide, doxorubicin, and rituximab; HR, hazard ratio; IHC, immunohistochemistry; IPI, International Prognostic Index; LDH, lactate dehydrogenase; R-CHOP, rituximab, cyclophosphamide, doxorubicin, vincristine, and prednisone.

Since initiation of Alliance/CALGB 50303, an NCl phase II study of DA-EPOCH-R in PMBCL reported an EFS rate of $93 \%$ in 51 patients with a median 5 years of follow-up. ${ }^{17} \mathrm{~A}$ retrospective, multicenter study reported an 86\% 3-year EFS rate in 156 patients with PMBCL receiving DA-EPOCH$\mathrm{R}^{18}$ In a second retrospective study of PMBCL, DA-EP$\mathrm{OCH}-\mathrm{R}(\mathrm{n}=76$ patients) and $\mathrm{R}-\mathrm{CHOP}(\mathrm{n}=56$ patients $)$ had 2-year PFS rates of $85 \%$ and $76 \%$, respectively $(P=.28) .{ }^{19}$ Consolidative RT was given to $59 \%$ of patients receiving
R-CHOP. There was no statistical difference $(P=.38)$ in 2-year PFS rate between the R-CHOP plus RT group (95\%) and $\mathrm{R}-\mathrm{CHOP}$ alone (88\%). Alliance/CALGB 50303 registered only 35 patients with $\mathrm{PMBCL}$, possibly reflecting investigator concern with randomization to R-CHOP without radiation. There was no difference in outcomes in this small subset.

Patients with DLBCL with $M Y C$-rearrangement $\left(M Y C^{+}\right)$, especially those with concomitant $B C L 2$ or $B C L 6$ 
TABLE 3. Selected Treatment-Related Toxicities That Differed by Treatment Arm Treatment

\begin{tabular}{|c|c|c|c|c|}
\hline \multirow[b]{2}{*}{ CTCAE Toxicity Grade } & \multicolumn{2}{|c|}{ Treatment } & \multirow[b]{2}{*}{$\begin{array}{c}\text { Total } \\
(\mathrm{n}=480)^{*}\end{array}$} & \multirow[b]{2}{*}{$P \dagger$} \\
\hline & $\begin{array}{c}\text { R-CHOP } \\
(\mathrm{n}=243)^{*}\end{array}$ & $\begin{array}{l}\text { DA-EPOCH-R } \\
(\mathrm{n}=237)^{*}\end{array}$ & & \\
\hline $3 / 4 / 5 \mathrm{AE}$ & & & & $<.001$ \\
\hline No & $53(21.8)$ & $4(1.7)$ & 57 (11.9) & \\
\hline Yes & $190(78.2)$ & $233(98.3)$ & $423(88.1)$ & \\
\hline 3/4 Hematologic & & & & $<.001$ \\
\hline No & $64(26.3)$ & $6(2.5)$ & $70(14.6)$ & \\
\hline Yes & $179(73.7)$ & $231(97.5)$ & $410(85.4)$ & \\
\hline 3/4 Nonhematologic & & & & $<.001$ \\
\hline No & $138(56.8)$ & $66(27.8)$ & $204(42.5)$ & \\
\hline Yes & $105(43.2)$ & $171(72.2)$ & $276(57.5)$ & \\
\hline 3/4 Infection & & & & .0494 \\
\hline No & $217(89.3)$ & $197(83.1)$ & $414(86.3)$ & \\
\hline Yes & $26(10.7)$ & $40(16.9)$ & $66(13.8)$ & \\
\hline 3/4 Febrile neutropenia & & & & $<.001$ \\
\hline No & $200(82.3)$ & $154(65.0)$ & $354(73.8)$ & \\
\hline Yes & $43(17.7)$ & $83(35.0)$ & $126(26.3)$ & \\
\hline 3/4 Mucositis & & & & .0017 \\
\hline No & $238(97.9)$ & 217 (91.6) & $455(94.8)$ & \\
\hline Yes & $5(2.1)$ & $20(8.4)$ & $25(5.2)$ & \\
\hline 1/2 Neuropathy & & & & $<.001$ \\
\hline No & $128(52.7)$ & $82(34.6)$ & $210(43.8)$ & \\
\hline Yes & $115(47.3)$ & $155(65.4)$ & $270(56.3)$ & \\
\hline 3/4 Neuropathy & & & & $<.001$ \\
\hline No & 235 (96.7) & $193(81.4)$ & $428(89.2)$ & \\
\hline Yes & $8(3.3)$ & $44(18.6)$ & 52 (10.8) & \\
\hline
\end{tabular}

NOTE. Data reported as No. (\%) unless otherwise indicated.

Abbreviations: AE, adverse event; CTCAE, National Cancer Institute Common Terminology Criteria for Adverse Events, version 4.0; DA-EPOCH-R, dose-adjusted etoposide, prednisone, vincristine, cyclophosphamide, doxorubicin, and rituximab; $\mathrm{R}-\mathrm{CHOP}$, rituximab, cyclophosphamide, doxorubicin, vincristine, and prednisone.

*Excludes 10 patients who did not initiate treatment and one patient in the R-CHOP arm without toxicity data submitted.

$\dagger \chi^{2} P$ value.

rearrangements (double hit), have a worse prognosis; the British Columbia Cancer Agency reported a 5-year PFS rate

\section{AFFILIATIONS}

${ }^{1}$ Washington University School of Medicine, St Louis, MO

${ }^{2}$ National Cancer Institute, National Institutes of Health, Bethesda, MD

${ }^{3}$ Duke University, Durham, NC

${ }^{4}$ Cleveland Clinic, Cleveland, $\mathrm{OH}$

${ }^{5}$ Mayo Clinic, Rochester, MN

${ }^{6}$ MedStar Georgetown University Hospital, Washington, DC

${ }^{7}$ University of Rochester, Rochester, NY

${ }^{8}$ The Ohio State University Comprehensive Cancer Center, Columbus, $\mathrm{OH}$

${ }^{9}$ Massachusetts General Hospital Cancer Center, Boston, MA

${ }^{10}$ Vanderbilt University Medical Center, Nashville, TN

${ }^{11}$ Northwestern University, Chicago, IL
}

of $31 \%$ with R-CHOP for the $8.8 \%$ of patients with $\mathrm{MYC}^{+}$ DLBCL versus $66 \%$ in the $M Y C^{-}$cohort $(P=.006){ }^{20} \mathrm{In}$ marked contrast, results of a multicenter phase II study of DA-EPOCH-R in 53 patients with $\mathrm{MYC}^{+}$DLBCL demonstrated a 4 -year EFS rate of $71 \%$ in all patients and $73.4 \%$ for patients with double-hit DLBCL. ${ }^{21}$ Coexpression of MYC and BCL2 proteins in DLBCL has also been associated with poor outcomes..$^{22-25}$ In our study, the lower incidence of the DE phenotype (15.6\%) compared with the literature again likely reflects a more favorable patient population. ${ }^{26}$ We found no differences by treatment arm in the MYC rearranged or patients with the DE phenotype, though, again, this subset was of insufficient size for statistical comparison. On the basis of encouraging phase II results with DA-EPOCH-R in patients with double-hit and Burkitt lymphoma compared with historical R-CHOP data, the results of Alliance/CALGB 50303 should not discourage use of DA-EPOCH-R in these settings. ${ }^{21,22}$

In summary, the primary end point of PFS was not different among patients treated with DA-EPOCH-R compared with those treated with standard R-CHOP. Unfortunately, those with the greatest unmet need are not well represented. Whether patients with high-risk IPI, high-grade double-hit and DE lymphomas, or PMBCL may benefit from DAEPOCH-R cannot be answered from our study, because of the subset size and statistical caveats. These limitations reflect challenges in current clinical trial design. ${ }^{16,27}$

We now understand DLBCL is even more heterogeneous than appreciated when this trial was designed. Recent work on the genetic drivers as well as the exome and transcriptome of DLBCL highlights its heterogeneity. ${ }^{28-30}$ Therefore, the National Clinical Trials Network is planning a precision medicine approach to identify molecular subsets of DLBCL and determine if specific chemotherapy platforms and/or targeted agents offer differential benefit. ${ }^{31}$ A primary focus is on patients with translocated or expressed BCL2/MYC who are treated with the BCL2 inhibitor venetoclax, with either R-CHOP or DA-EPOCH-R, depending upon FISH and DE status. Future studies may benefit from steps allowing more rapid initiation of treatment, such as prephase or permitted standard cycle 1 therapy, thus facilitating inclusion of high-risk patients.

\footnotetext{
${ }^{12}$ University of Wisconsin, Madison, WI

${ }^{13}$ Univeristy of Washington, Seattle, WA

${ }^{14}$ Cornell University Medical College, New York, NY

${ }^{15}$ Fox Chase Cancer Center, Philadelphia, PA

${ }^{16}$ University of North Carolina, Chapel Hill, NC

${ }^{17}$ Memorial Sloan Kettering Cancer Center, New York, NY
}

\section{CORRESPONDING AUTHOR}

Nancy L. Bartlett, MD, Washington University School of Medicine, Campus Box 8056, St Louis, MO 63110; e-mail: nbartlet@wustl.edu. 


\section{PRIOR PRESENTATION}

Presented at the American Society of Hematology Annual Meeting, San Diego, CA, December 3-6, 2016.

\section{SUPPORT}

Supported by the National Cancer Institute of the National Institutes of Health (Grants No. U10CA180821 and U10CA180882 (to the Alliance for Clinical Trials in Oncology; and U10CA180847, U10CA180833, U10CA180799 and U10CA180820).

\section{AUTHOR CONTRIBUTIONS}

Conception and design: Wyndham H. Wilson, Sin-Ho Jung, Bruce D. Cheson, Louis M. Staudt, Richard I. Fisher, Heiko Schöder, Andrew D. Zelenetz

Financial support: Wyndham $\mathrm{H}$. Wilson

Administrative support: Wyndham H. Wilson, Amy Chadburn, John P. Leonard

Provision of study materials or patients: Nancy L. Bartlett, Wyndham H. Wilson, Bruce D. Cheson, Brad S. Kahl, Jonathan W. Friedberg, Nina D. Wagner-Johnston, Kristie A. Blum, Jeremy S. Abramson, Nishitha M. Reddy, Jane N. Winter, Julie E. Chang, Ajay K. Gopal, John P. Leonard
Collection and assembly of data: Nancy L. Bartlett, Wyndham H. Wilson, Sin-Ho Jung, Eric D. Hsi, Matthew J. Maurer, Levi D. Pederson, Mei-Yin C. Polley, Brandelyn N. Pitcher, Brad S. Kahl, Nina D. Wagner-Johnston, Kristie A. Blum, Jeremy S. Abramson, Nishitha M. Reddy, Julie E. Chang, Ajay K. Gopal, Amy Chadburn, Kristy L. Richards, Heiko Schöder, Andrew D. Zelenetz, John P. Leonard

Data analysis and interpretation: Nancy L. Bartlett, Wyndham H. Wilson, Sin-Ho Jung, Matthew J. Maurer, Levi D. Pederson, Mei-Yin C. Polley, Brandelyn N. Pitcher, Bruce D. Cheson, Brad S. Kahl, Jonathan W. Friedberg, Louis M. Staudt, Nina D. Wagner-Johnston, Kristie A. Blum, Jeremy S. Abramson; Nishitha M. Reddy, Jane N. Winter, Julie E. Chang, Ajay K. Gopal, Amy Chadburn, Susan Mathew, Heiko Schöder, Andrew D. Zelenetz, John P. Leonard

Manuscript writing: All authors

Final approval of manuscript: All authors

Accountable for all aspects of the work: All authors

\section{AUTHORS' DISCLOSURES OF POTENTIAL CONFLICTS OF INTEREST} AND DATA AVAILABILITY STATEMENT

Disclosures provided by the authors and data availability statement (if applicable) are available with this article at DOI https://doi.org/10.1200/ JCO.18.01994

\section{REFERENCES}

1. Coiffier B, Lepage E, Brière J, et al: CHOP chemotherapy plus rituximab compared with CHOP alone in elderly patients with diffuse large-B-cell lymphoma. N Engl J Med 346:235-242, 2002

2. Feugier $\mathrm{P}$, Van Hoof A, Sebban C, et al: Long-term results of the R-CHOP study in the treatment of elderly patients with diffuse large B-cell lymphoma: A study by the Groupe d'Etude des Lymphomes de l'Adulte. J Clin Oncol 23:4117-4126, 2005

3. Habermann TM, Weller EA, Morrison VA, et al: Rituximab-CHOP versus CHOP alone or with maintenance rituximab in older patients with diffuse large B-cell lymphoma. J Clin Oncol 24:3121-3127, 2006

4. Pfreundschuh M, Trümper L, Österborg A, et al: CHOP-like chemotherapy plus rituximab versus CHOP-like chemotherapy alone in young patients with goodprognosis diffuse large-B-cell lymphoma: A randomised controlled trial by the MabThera International Trial (MInT) Group. Lancet Oncol 7:379-391, 2006

5. Pfreundschuh M, Kuhnt E, Trümper L, et al: CHOP-like chemotherapy with or without rituximab in young patients with good-prognosis diffuse large-B-cell lymphoma: 6-Year results of an open-label randomised study of the MabThera International Trial (MInT) Group. Lancet Oncol 12:1013-1022, 2011

6. Crump M, Neelapu SS, Farooq U, et al: Outcomes in refractory diffuse large B-cell lymphoma: Results from the international SCHOLAR-1 study. Blood 130: 1800-1808, 2017

7. Gutierrez M, Chabner BA, Pearson D, et al: Role of a doxorubicin-containing regimen in relapsed and resistant lymphomas: An 8-year follow-up study of EPOCH. J Clin Oncol 18:3633-3642, 2000

8. Wilson WH, Grossbard ML, Pittaluga S, et al: Dose-adjusted EPOCH chemotherapy for untreated large B-cell lymphomas: A pharmacodynamic approach with high efficacy. Blood 99:2685-2693, 2002

9. Wilson WH, Gutierrez M, O'Connor P, et al: The role of rituximab and chemotherapy in aggressive b-cell lymphoma: A preliminary report of dose-adjusted EPOCH-R. Semin Oncol 29:41-47, 2002 (suppl 2)

10. Wilson WH, Dunleavy K, Pittaluga S, et al: Phase II study of dose-adjusted EPOCH and rituximab in untreated diffuse large B-cell lymphoma with analysis of germinal center and post-germinal center biomarkers. J Clin Oncol 26:2717-2724, 2008

11. Wilson WH, Jung SH, Porcu P, et al: A Cancer and Leukemia Group B multi-center study of DA-EPOCH-rituximab in untreated diffuse large B-cell lymphoma with analysis of outcome by molecular subtype. Haematologica 97:758-765, 2012

12. Cheson BD, Horning SJ, Coiffier B, et al: Report of an international workshop to standardize response criteria for non-Hodgkin's lymphomas. J Clin Oncol 17: 1244-1253, 1999

13. Cerhan JR, Link BK, Habermann TM, et al: Cohort profile: The Lymphoma Specialized Program of Research Excellence (SPORE) Molecular Epidemiology Resource (MER) cohort study. Int J Epidemiol 46:1753-1754i, 2017

14. Maurer MJ, Ghesquières H, Link BK, et al: Diagnosis-to-treatment interval is an important clinical factor in newly diagnosed diffuse large B-cell lymphoma and has implication for bias in clinical trials. J Clin Oncol 36:1603-1610, 2018

15. Leonard JP, Kolibaba KS, Reeves JA, et al: Randomized phase II study of R-CHOP with or without bortezomib in previously untreated patients with non-germinal center B-cell-like diffuse large B-cell lymphoma. J Clin Oncol 35:3538-3546, 2017

16. Goy A: Succeeding in breaking the R-CHOP ceiling in DLBCL: Learning from negative trials. J Clin Oncol 35:3519-3522, 2017

17. Dunleavy K, Pittaluga S, Maeda LS, et al: Dose-adjusted EPOCH-rituximab therapy in primary mediastinal B-cell lymphoma. N Engl J Med 368:1408-1416, 2013

18. Giulino-Roth L, O'Donohue T, Chen Z, et al: Outcomes of adults and children with primary mediastinal B-cell lymphoma treated with dose-adjusted EPOCH-R. Br J Haematol 179:739-747, 2017

19. Shah NN, Szabo A, Huntington SF, et al: R-CHOP versus dose-adjusted R-EPOCH in frontline management of primary mediastinal B-cell lymphoma: a multicentre analysis. Br J Haematol 180:534-544, 2018

20. Savage KJ, Johnson NA, Ben-Neriah S, et al: MYC gene rearrangements are associated with a poor prognosis in diffuse large B-cell lymphoma patients treated with R-CHOP chemotherapy. Blood 114:3533-3537, 2009 
21. Dunleavy K, Fanale MA, Abramson JS, et al: Dose-adjusted EPOCH-R (etoposide, prednisone, vincristine, cyclophosphamide, doxorubicin, and rituximab) in untreated aggressive diffuse large B-cell lymphoma with MYC rearrangement: A prospective, multicentre, single-arm phase 2 study. Lancet Haematol 5: e609-e617, 2018

22. Hu S, Xu-Monette ZY, Tzankov A, et al: MYC/BCL2 protein coexpression contributes to the inferior survival of activated B-cell subtype of diffuse large B-cell lymphoma and demonstrates high-risk gene expression signatures: A report from The International DLBCL Rituximab-CHOP Consortium Program. Blood 121: 4021-4031, 2013

23. Perry AM, Alvarado-Bernal Y, Laurini JA, et al: MYC and BCL2 protein expression predicts survival in patients with diffuse large B-cell lymphoma treated with rituximab. Br J Haematol 165:382-391, 2014

24. Herrera AF, Mei M, Low L, et al: Relapsed or refractory double-expressor and double-hit lymphomas have inferior progression-free survival after autologous stem-cell transplantation. J Clin Oncol 35:24-31, 2017

25. Savage KJ, Slack GW, Mottok A, et al: Impact of dual expression of MYC and BCL2 by immunohistochemistry on the risk of CNS relapse in DLBCL. Blood 127 : 2182-2188, 2016

26. Karube K, Campo E: MYC alterations in diffuse large B-cell lymphomas. Semin Hematol 52:97-106, 2015

27. Polley MYC, Freidlin B, Korn EL, et al: Statistical and practical considerations for clinical evaluation of predictive biomarkers. J Natl Cancer Inst 105:1677-1683, 2013

28. Reddy A, Zhang J, Davis NS, et al: Genetic and functional drivers of diffuse large B cell lymphoma. Cell 171:481-494.e15, 2017

29. Chapuy B, Stewart C, Dunford AJ, et al: Molecular subtypes of diffuse large B cell lymphoma are associated with distinct pathogenic mechanisms and outcomes. Nat Med 24:679-690, 2018 Erratum: Nat Med 24:1290-1291,1292, 2018

30. Schmitz R, Wright GW, Huang DW, et al: Genetics and pathogenesis of diffuse large B-cell lymphoma. N Engl J Med 378:1396-1407, 2018

31. Nowakowski GS, Blum KA, Kahl BS, et al: Beyond RCHOP: A blueprint for diffuse large B cell lymphoma research. J Natl Cancer Inst 108:djw257, 2016

\section{You've Guided Them Through Treatment: What's Next?}

As a supplement to the guidance ASCO offers on survivorship care, the ASCO Cancer Survivorship Compendium serves as a repository of tools and resources to enable oncology providers to implement or improve survivorship care within their practices. Delivering high-quality survivorship care can enhance patients' long-term health by complementing efforts to manage concerns related to cancer treatment and survivorship.

To view the Compendium's online resources to support your own survivorship care efforts, visit asco.org/survivorship 
Dose-Adjusted EPOCH-R Compared With R-CHOP as Frontline Therapy for Diffuse Large B-Cell Lymphoma: Clinical Outcomes of the Phase III Intergroup Trial Alliance/CALGB 50303

The following represents disclosure information provided by authors of this manuscript. All relationships are considered compensated. Relationships are self-held unless noted. I = Immediate Family Member, Inst = My Institution. Relationships may not relate to the subject matter of this manuscript. For more information about ASCO's conflict of interest policy, please refer to www.asco.org/rwc or ascopubs.org/jco/site/ifc.

\section{Nancy L. Bartlett}

Consulting or Advisory Role: Pfizer, Seattle Genetics, ADC Therapeutics Research Funding: Seattle Genetics (Inst), Genentech (Inst), Kite Pharma (Inst), Merck (Inst), Bristol-Myers Squibb (Inst), Celgene (Inst), Immune Design (Inst), Forty Seven (Inst), Affimed Therapeutics (Inst), Janssen (Inst), Pharmacyclics (Inst), Millennium (Inst), Gilead Sciences (Inst)

Eric D. Hsi

Honoraria: Seattle Genetics, Celgene, Jazz Pharmaceuticals Research Funding: Eli Lilly (Inst), AbbVie (Inst), Cellerant Therapeutics (Inst)

\section{Matthew J. Maure}

Consulting or Advisory Role: MorphoSys

Research Funding: Celgene (Inst), NanoString Technologies (Inst)

Bruce D. Cheson

Consulting or Advisory Role: Acerta Pharma, TG Therapeutics, Bayer, Roche, AbbVie, Pharmacyclics/Janssen, Morphosys, Sunesis Pharmaceuticals, Celgene

Research Funding: Roche (Inst), Acerta Pharma (Inst), Gilead Sciences (Inst), AbbVie (Inst), TG Therapeutics (Inst)

\section{Brad S. Kahl}

Consulting or Advisory Role: Celgene, Juno Therapeutics, AbbVie, Pharmacyclics, Acerta Pharma, ADC Therapeutics, Pharmacyclics, Genentech, Roche

Research Funding: Genentech (Inst), Acerta Pharma (Inst), ADC Therapeutics (Inst)

Travel, Accommodations, Expenses: Celgene, Juno Therapeutics, Genentech Roche, AbbVie, Millennium, Seattle Genetics

\section{Jonathan W. Friedberg}

Consulting or Advisory Role: Bayer, Astellas Pharma

Research Funding: Seattle Genetics (Inst), Kite Pharma (Inst)

Patents, Royalties, Other Intellectual Property: Patient on bone marrow

microenvironment signals (I)

Travel, Accommodations, Expenses: Roche

Louis M. Staudt

Patents, Royalties, Other Intellectual Property: Patents and patents pending regarding gene expression profiling in lymphoma that have been licensed by Nanostring and for which I receive royalties.

Nina D. Wagner-Johnston

Consulting or Advisory Role: Juno Therapeutics, ADC Therapeutics, Janssen Oncology, Gilead Sciences

Research Funding: Merck, Novartis/Pfizer, Genentech, Astex Pharmaceuticals

\section{Kristie A. Blum}

Research Funding: MorphoSys, Merck, Bristol-Myers Squibb, Celgene, Novartis, Janssen

\section{Jeremy S. Abramson}

Consulting or Advisory Role: Gilead Sciences, Seattle Genetics, Celgene, Novartis, Amgen, Juno Therapeutics, Karyopharm Therapeutics, Verastem, Bayer, AbbVie, Janssen, Merck, Kite Pharma, Genentech, EMD Serono Research Funding: Seattle Genetics (Inst), Celgene (Inst), Genentech (Inst), Juno Therapeutics (Inst), Al Therapeutics (Inst)

\section{Nishitha M. Reddy}

Consulting or Advisory Role: Celgene, AbbVie, Bristol-Myers Squibb, Adaptive Biotechnologies

Speakers' Bureau: Gilead Science

Research Funding: Bristol-Myers Squibb (Inst)

\section{Jane N. Winter}

Consulting or Advisory Role: Novartis (I), Ariad (I), CVS Caremark (I), Celgene (I), Amgen (I), Boston Biomedicine Pharma (I), AbbVie (I), DAVAOncology (I),

Adicet Bio, Bayer, Merck, Gilead Sciences, Janssen

Research Funding: Janssen Oncology (Inst), GlaxoSmithKline (Inst), Astellas Pharma (Inst), ERYTECH Pharma (Inst), Celgene (I), Daiichi Sankyo (I), Merck, Curis

Patents, Royalties, Other Intellectual Property: Royalties from UptoDate (I)

Travel, Accommodations, Expenses: Merck, Bayer, Adicet Bio, Gilead

Sciences, Janssen

\section{Julie E. Chang}

Research Funding: Celgene, Roche, Adaptive Biotechnologies

Ajay K. Gopal

Stock and Other Ownership Interests: Compliment Corp

Honoraria: Amgen, Takeda, Sanofi, Gilead Sciences, Janssen, Acerta Pharma/ AstraZeneca, Seattle Genetics

Consulting or Advisory Role: Amgen, Janssen, Sanofi, Gilead Sciences, Takeda, Seattle Genetics

Research Funding: Janssen (Inst), Teva (Inst), Gilead Sciences (Inst), Merck (Inst), Seattle Genetics (Inst), Bristol-Myers Squibb (Inst), Pfizer (Inst), eFFECTOR Therapeutics (Inst), Spectrum Pharmaceuticals (Inst), Takeda (Inst) Travel, Accommodations, Expenses: Amgen, Acerta Pharma/AstraZeneca

\section{Amy Chadburn}

Honoraria: Imedex

Richard I. Fisher

Consulting or Advisory Role: Pharmacyclics/Janssen, Roche, Kite Pharma, Seattle Genetics, Sandoz, Celgene, Genentech, Bayer, AstraZeneca, Adaptive Biotechnologies, Ion Solutions

Expert Testimony: Roche

Heiko Schöder

Consulting or Advisory Role: Aileron Therapeutics

\section{Andrew D. Zelenetz}

Stock and Other Ownership Interests: Adaptive Biotechnologies Honoraria: National Comprehensive Cancer Network, Clinical Care Options, PER, Oncology Information Group

Consulting or Advisory Role: Gilead Sciences, Amgen, Roche, Celgene, Medscape, AstraZeneca, DAVAOncology, MorphoSys, BeiGene, Cancer Support Community, Janssen, MEI Pharma, Novartis, Pfizer, Prime Education, TRM Oncology, Vaniam Group, Verastem, Pharmacyclics, Karyopharm Therapeutics Research Funding: Roche, Gilead Sciences, MEI Pharma, BeiGene

John P. Leonard

Consulting or Advisory Role: Celgene, Biotest, Sunesis Pharmaceuticals, BristolMyers Squibb, Gilead Sciences, Epizyme, Pfizer, Bayer, Roche, ADC Therapeutics, MEI Pharma, AstraZeneca, Novartis, Merck, Sutter Medical Group, Morphosys, Beigene, Nordic Nanovector, Bristol-Myers Squibb, United Therapeutics, Karyopharm Therapeutics, Sandoz

Research Funding: Celgene (Inst), Alliance for Clinical Trials in Oncology (Inst), Takeda (Inst), Pfizer (Inst), National Cancer Institute (Inst)

Travel, Accommodations, Expenses: BeiGene

No other potential conflicts of interest were reported. 